\title{
Caracterizacion de la Comunicación Ulnar-Mediano Palmar Profunda en una Muestra con Material Cadavérico
}

\author{
Characterization of Ulnar-Median Deep Palmar \\ Communication in a Sample with Cadaveric Material
}

Luis Ernesto Ballesteros Acuña ${ }^{1}$; Pedro Luis Forero Porras ${ }^{2,3}$ \& Oscar Javier Larrotta Rojas ${ }^{4}$

BALLESTEROS, A. L. E.; FORERO, P. P. L. \& LARROTTA, R. O. J. Caracterizacion de la comunicación ulnar-mediano palmar profunda en una muestra con material cadaverico. Int. J. Morphol., 38(5):1192-1196, 2020.

RESUMEN:La comunicación Ulnar-Mediano Palmar Profunda (CUMPP) es la conexión entre la rama profunda del nervio ulnar (NU) y la rama del nervio mediano (NM) que inerva a los músculos tenares como la cabeza superficial del flexor corto del pulgar. Son escasos los trabajos que se ocupan de esta rama comunicante, y su prevalencia es reportada con una amplia variabilidad, en un rango del 16-77 \%. Este estudio no probabilístico, descriptivo, transversal, evaluó la frecuencia y características morfológicas de la CUMPP en 106 manos de especímenes cadavéricos frescos no reclamados, a quienes se les practicó necropsia en el Instituto de Medicina Legal de Bucaramanga (Colombia). Se observó rama comunicante CUMPP en 39 especímenes (50,2 \%), de los cuales 12 (44,5\%) fueron bilaterales, $15(55,6 \%)$ unilaterales, con predominio unilateral derecho para 9 casos (60\%), sobre el izquierdo de tan solo 6 casos (40\%). No se evidenciaron diferencias estadísticamente significativas con relación al lado de presentación ( $\mathrm{P}=0,223)$. En 21 especímenes (54 \%) se observó el tipo IV; mientras que el tipo I fue encontrado en 4 casos (10\%). El promedio de la longitud de la rama comunicante fue de 24,67 DE 6,46 mm; mientras que la distancia del punto proximal de la CUMPP al surco distal del carpo fue de 41,4 DE 2,6 mm. Nuestros hallazgos no son concordantes con lo reportado en la mayoría de estudios previos. Diversos factores como el tamaño de las muestras, las diferentes metodologías de medición y las expresiones fenotípicas de cada grupo de población evaluado, pueden explicar la variabilidad de la CUMPP.

PALABRAS CLAVE: Nervio Mediano (NM); Nervio Ulnar (NU); Ramo profunda nervio ulnar; Región palmar profunda; Ramo comunicante.

\section{INTRODUCCIÓN}

La comunicación Ulnar-Mediano Palmar Profunda (CUMPP) fue descrita por Riche y por Cannieu en 1887 como la conexión entre el ramo profundo del nervio ulnar (NU) que inerva la cabeza profunda del flexor corto del pulgar y el ramo del nervio mediano (NM) que inerva la cabeza superficial del flexor corto del pulgar (Harness \& Sekeles, 1971).

La CUMPP no es descrita de manera explícita en los textos de anatomía, aunque si se menciona sobre la inervación del flexor corto del pulgar por parte de ramos del NM y del NU (Kaplan et al., 1948; Lippert et al., 2006; Moore et al., 2010; Drake et al., 2010). Son escasos los trabajos que se ocupan del ramo comunicante, y su prevalencia es reportada con una amplia variabilidad, en un rango del 16-77 \% (Harness \& Sekeles; Loukas et al., 2011; Brown \& Landau, 2013).
La doble inervación de algunos músculos del tenar que se presenta cuando existe esta ansa comunicante se puede convertir en factor de protección en caso de lesiones del NM o de NU, encontrándose parálisis parciales del flexor corto del pulgar cuando alguna de estos ramos terminales del plexo braquial ha sufrido lesiones (Higuet et al., 1943), durante procedimientos quirúrgicos y dificultar el estudio electrofisiológico en la detección de neuropatías (Caetano et al., 2019). El ramo CUMPP ha sido descrito por métodos anatómicos, clínicos y electrofisiológicos en otros grupos poblacionales de manera poco detallada y con predominio en sus características cualitativas (Brown \& Landau). Teniendo en cuenta que el factor étnico es decisivo para la presencia de diferentes expresiones anatómicas y la escasa información sobre la CUMPP, hizo pertinente llevar a cabo este estudio en material cadavérico

\footnotetext{
${ }^{1}$ Departamento de Ciencias Básicas, Facultad de Salud, Universidad Industrial de Santander. Bucaramanga, Colombia.

${ }^{2}$ Departamento de Patología, Facultad de Salud, Universidad Industrial de Santander. Bucaramanga, Colombia.

${ }^{3}$ Instituto Nacional de Medicina Legal y Ciencias Forenses. Bucaramanga, Colombia.

${ }^{4}$ Facultad de Salud, Universidad Industrial de Santander, Colombia.
} 
fresco en una muestra de población mestiza predominantemente en América Latina, con el fin de obtener información propia de referencia.

\section{MATERIAL Y MÉTODO}

Este estudio no probabilístico, descriptivo transversal evaluó la frecuencia y características morfológicas de la CUMPP en 106 manos de especímenes cadavéricos frescos no reclamados, a quienes se les practicó necropsia en el Instituto de Medicina Legal de Bucaramanga (Colombia).La muestra cumplió con los siguientes criterios de inclusión: sujetos masculinos, adultos (mayores de 18 años), del grupo étnico mestizo (mezcla de blancos-hispánicos e indígenas), sin evidencia de traumatismo directo o de patologías que comprometiera la región anatómica a estudio.

Cada región palmar, se abordó mediante incisión mediana, desde el surco distal del carpo hasta el pliegue metacarpo falángico del tercer dedo, que comprometió piel, tejido celular subcutáneo y aponeurosis palmar, los cuales fueron reclinados hacia medial y lateralmente, quedando expuestos los tendones flexores. Posteriormente se disecó el NU a nivel del surco ulnar del carpo identificándose sus ramos superficial y profundo. Luego, se practicó incisión mediana del retináculo flexor, disecándose el ramo tenar y digitales del NM.

En la región palmar profunda, luego de la liberación de los tendones flexores superficiales y profundos, mediante la utilización de lupas con magnificación " 4 x", se identificó el ramo profundo del NU y en los casos que presentaran conexiones al NM, se caracterizó los ramos comunican- tes, tipificándolos de acuerdo a los criterios de Harness \& Sekeles.

Tipo I: un ramo comunicante entre el ramo para el aductor del pulgar y el ramo del mediano para la cabeza superficial del flexor corto del pulgar.

Tipo II: entre el ramo para el aductor del pulgar y el nervio digital del pulgar.

Tipo III: una rama comunicante entre el ramo para el aductor del pulgar y el ramo digital del pulgar.

Tipo IV, una comunicación entre el ramo para la cabeza profunda del flexor corto del pulgar y el ramo digital del dedo índice.

En los casos positivos para CUMPP, se midió la longitud de la rama, y la distancia del punto proximal y distal de la rama, con relación a la parte media surco distal del carpo.

Para cada una de las muestras se realizaron registros morfo-métricos con calibrador digital (Mitotuyo $®)$, y los hallazgos fueron fotografiados, con cámara Cannon T2I. Luego de registrarse los hallazgos macroscópicos, los ramos comunicantes fueron resecados, sometidos a fijación con formaldehido al $10 \%$, coloreados con hematoxilina-eosina y evaluados al microscopio con el fin de confirmar o descartar su naturaleza de estructura nerviosa.

Los hallazgos obtenidos fueron almacenados en Excel y los análisis estadísticos se realizaron en EPI-INFO y SigmaStat. Las variables continuas se describieron por medio de medidas de resumen estadístico: promedio, desviación estándar; las variables nominales con sus proporciones. Se realizaron las pruebas estadísticas de chi (X2) cuadrado, ANOVA y test-t, aceptando un error alfa hasta de un $5 \%$.
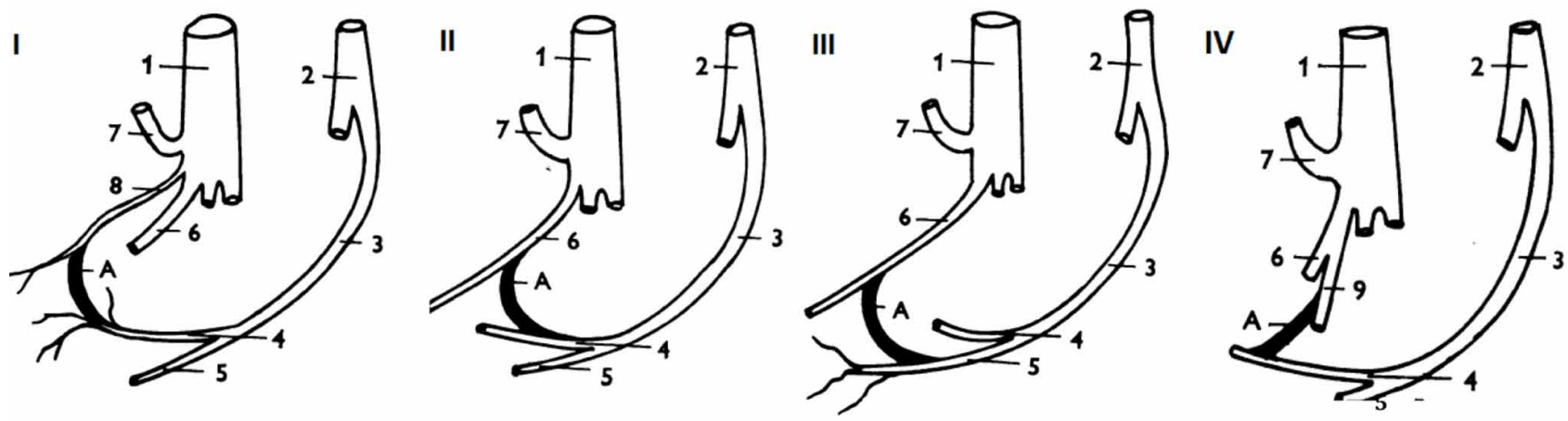

Fig. 1 Esquema que representa los diversos tipos de comunicación entre el NM y la rama profunda de NU. Tomado y modificado de: Harness D, Sekeles E. The double anastomotic innervation of thenar muscles. J. Anat., 109(3):461-6, 1971. 1. Nervio mediano; 2. Nervio ulnar, 3: Rama profunda del nervio ulnar; 4. Ramo para la cabeza profunda del flexor corto del pulgar, 5, Ramo para el aductor del pulgar; 6. Rama digital del pulgar; 7. Rama recurrente, 8 . Ramo del nervio mediano para la cabeza superficial del pulgar; 9. Ramo digital para el dedo índice. 


\section{RESULTADOS}

Se observó un ramo comunicante CUMPP en 39 especímenes $(50,2 \%)$, de los cuales $12(44,5 \%)$ fueron bilaterales, $15(55,6 \%)$ unilaterales, con predominio unilateral derecho 9 casos (60\%), sobre el izquierdo 6 (40\%). No se evidenciarondiferencias estadísticamente significativas con relación al lado de presentación $(\mathrm{P}=0,223)$.

De acuerdo a la clasificación de Harness \& Sekeles se observó el Tipo IV en 21 especímenes (54\%), Tipo II y Tipo III cada uno en siete casos respectivamente (18\%);

Fig. 2. Comunicación ulnar-mediano palmar profunda. Vista anterior mano derecha. ABP. Músculo abductor corto del pulgar; FCP. Músculo flexor corto del pulgar; AP. Músculo aductor del pulgar; RF. Retináculo flexor; TC. Túnel del carpo; TEN. Tenar; 1. Primer nervio digital, ramo del mediano; 2. Rama profunda del Nervio ulnar; $(\bullet)$ ramo comunicante entre la rama profunda del ulnar y la rama recurrente del nervio mediano.

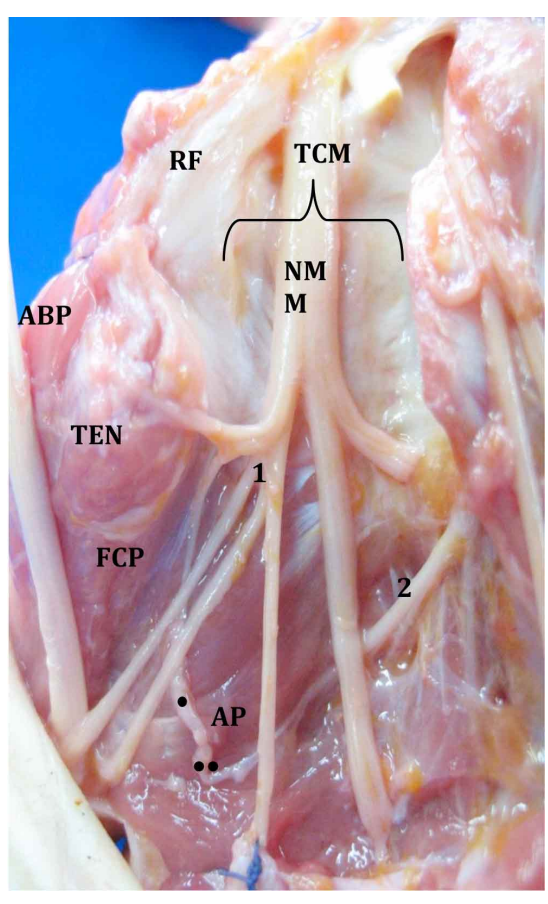

mientras que el Tipo I se encontró en cuatro casos (10 \%). (Fig. 2). El promedio de la longitud del ramo comunicante fue de 24,67 DE 6,46 mm; mientras que la distancia del punto proximal del ramo comunicante al surco distal del carpo fue de 41,4 DE 2,6 mm y de su punto distal al surco referente fue de 45,8 DE 2,56 mm (Fig. 3).

Se observó al microscopio que el $100 \%$ de los ramos comunicantes, correspondían a estructuras nerviosas de nervio periférico, con tejido conectivo epineuro y los fascículos rodeados de perineuro (Fig. 4).

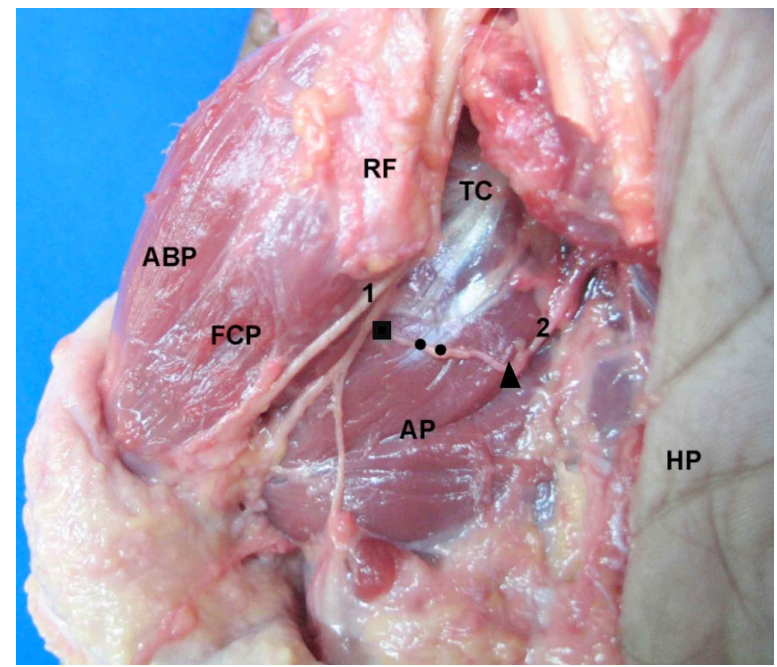

Fig. 3. Comunicación ulnar-mediano palmar profunda Tipo III. Vista anterior mano derecha. (Se ha resecado los tendones flexores) FCP. Músculo flexor corto del pulgar; AP. Músculo aductor del pulgar; TC túnel carpo; HP. Hipotenar; 1. Primer nervio digital, ramo del mediano; 2. Ramo profundo del Nervio ulnar; $(\bullet)$ ramo comunicante entre el ramo profundo del ulnar y el primer nervio digital; $\Delta$ Punto Proximal del ramo; - Punto distal del ramo comunicante.
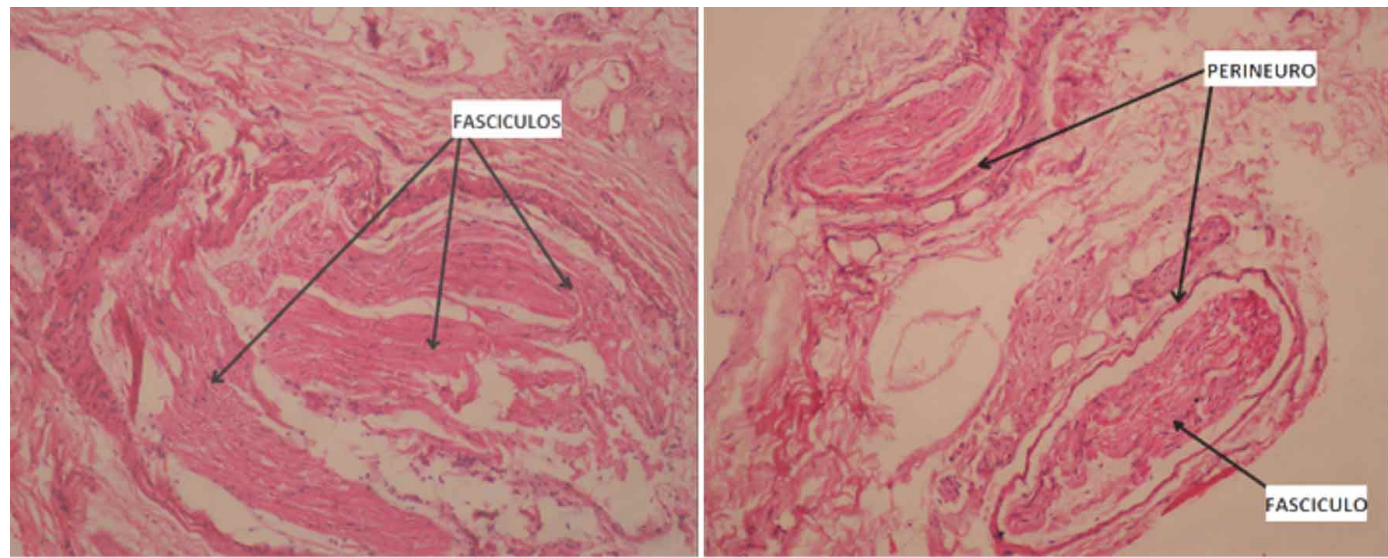

Fig. 4. Corte transversal de una anastomosis de ulnar-mediano palmar profunda. (Magnificación 10X, coloración hematoxilina-eosina.) $(\rightarrow$ ) Las fechas señalan varios fascículos o haces nerviosos rodeados de tejido conectivo, y el tejido conectivo perineural. 
BALLESTEROS, A. L. E.; FORERO, P. P. L. \& LARROTTA, R. O. J. Caracterizacion de la comunicación ulnar-mediano palmar profunda en una muestra con material cadaverico Int. J. Morphol., 38(5):1192-1196, 2020.

\section{DISCUSION}

Se describe una prevalencia CUMPP del 50,2\%, cifra que es superior al $16 \%$ reportado por Loukas et al., y muy inferior al $77 \%$ informado por Harness \& Sekeles. El predominio unilateral $(55,6 \%)$ sobre el bilateral en la muestra observada, y del lado derecho, aunque sin significancia estadística, es concordante con los reportes previos. En los hallazgos de Harness \& Sekeles, el tipo I aparece con una frecuencia de $52 \%$, mientras que el resto de casos correspondieron a los demás tipos, sin especificar frecuencias: Cannieu, citado por Harness \& Sekeles encontraron el ramo comunicante Tipo III en el $15 \%$ de los 20 manos que disecó. Loukas et al. no tienen en cuenta las previas caracterizaciones y limita su reporte al señalar que, en todos los casos, el ramo comunicante se desprendía del ramo profundo del ulnar y conectaba con el mediano en el punto previo a la emergencia de sus ramos digitales.

En nuestra serie, el tipo IV fue el mas frecuente, mientras que para Harness \& Sekeles de manera significativa fue el tipo I, lo cual nos permite deducir que el ramo comunicante puede conectar directamente ramos motores o utilizando los nervios digitales para el pulgar o el índice, el ramo profundo del NU contribuye a la inervación de los músculos del tenar o de los primeros músculos lumbricales compartiendo inervación con el NM. Diversos factores como el tamaño de las muestras, las diferentes metodologías de medición y las expresiones fenotípicas de cada grupo de población evaluado, pueden explicar la variabilidad de la CUMPP.

La importancia de la doble inervación de algunos músculos del tenar derivadas de la presencia de este ramo comunicante se orienta a que actúa como factor que minimiza la gravedad de las lesiones del NM y modifica la semiología funcional de la región tenariana (Tamagawa et al., 2004; Higuet et al.; Paraskevas et al., 2010). A pesar de que esta comunicación nerviosa es citada con frecuencia en el escenario clínico, la poca información existente de carácter anatómico amerita que se realicen nuevas investigaciones sobre esta estructura.

En estudios previos no se han reportado aspectos morfométricos de la CUMPP, por lo que los datos registrados en nuestra serie, permiten recrear en el segmento lateral y distal del tenar, la trayectoria de este ramo que cursa sobre la superficie del aductor del pulgar, tomando como referencia la distancia de los puntos proximal y distal del ramo al segmento medio del surco distal del carpo. Estos datos biométricos permiten un mejor diseño de los procedimientos quirúrgicos que se realicen en la región tenar y con ello se reduce los riesgos de lesionar el ramo comunicante y de alterar la fina biomecánica de esta región de la mano.

La exhaustiva revisión de la literatura realizada sobre las diversas conexiones existentes entre los ramos terminales del plexo braquial, arrojó que solo un trabajo (Shu et al., 1999) hizo la evaluación bajo las perspectivas macroscópica e histológica. El estudio microscópico de las ramas nerviosas, permite confirmar su carácter, al identificarse el tejido conectivo epineural, y los fascículos nerviosos rodeados del perineuro. Este procedimiento confirma los hallazgos y evita algún sesgo de los resultados. Es de Considerar que la verificación histológica de los ramos comunicantes, como se realizó en el presente trabajo, genera una mayor confiabilidad de los resultados, y su implementación debe acompañar el estudio de las fibras muy delgadas, que generan confusión o duda sobre si se trata de una banda de tejido conectivo o de una fibra nerviosa. Es probable que en muchos casos hayan sido reportados como conexiones nerviosas estructuras que no lo eran.

Estudios electrofisiológicos han mostrado que la estimulación del NM, suele producir una ligera respuesta sensitiva en el usual territorio del NU en la mano, lo cual podría interpretarse por la existencia de ramo comunicante. De igual manera, estimulaciones fisiológicas de NU en presencia de la variante, puede mostrar latencias normales en los registros electrofisiológicos de la zona tenar, caso contrario se ve en pacientes con denervación de la rama ulnar, donde se observa pérdida parcial de las funciones intrínsecas de la región tenar (Ahadi et al., 2016; Martin et al., 2019). En la posible distribución de fibras sensoriales en la CUMPP, los estudios electrofisiológicos referenciados no son concluyentes y no determinan modificación de los territorios sensoriales del NM y de NU a nivel palmar o digital.

Enfatizamos la importancia del conocimiento anatómico y biométrico de la CUMPP útil en el correcto planeamiento de procedimientos invasivos o quirúrgicos de la región tenar.

AGRADECIMIENTOS. Nuestra gratitud al Instituto Nacional de Medicina Legal y Ciencias Forenses por el suministro de las muestras anatómicas utilizadas en este estudio.

BALLESTEROS, A. L. E.; FORERO, P. P. L. \& LARROTTA, R. O. J. Characterization of ulnar-median deep palmar communication in a sample with cadaveric material. Int. J. Morphol., 38(5):1192-1196, 2020.

SUMMARY: Deep Palmar Ulnar-Medium Communication (DPUMC) is the connection between the deep 
branch of the ulnar nerve (UN) and the median nerve (MN) branch, that innervates the thenar muscles as the superficial head of the short flexor of the thumb. Few studies dealing with this communicating branch, and its prevalence is reported with a wide variability in the range of 16-77\%. This non-probabilistic, descriptive, cross-sectional study; evaluated the frequency and morphological characteristics of DPUMC in 106 hands of fresh unclaimed cadaveric specimens, that underwent necropsy at the Institute of Legal Medicine of Bucaramanga (Colombia). DPUMC communicating branch was observed in 39 specimens (50.2\%), of which $12(44.5 \%)$ were bilateral, $15(55.6 \%)$ unilateral, with right unilateral predominance for 9 cases $(60 \%)$, on the left of only 6 cases $(40 \%)$. There were no statistically significant differences in relation to the presentation side $(\mathrm{P}=0.223)$. In 21 specimens (54 $\%)$, type IV was observed; while type I was found in 4 cases (10 $\%)$. The average length of the communicating branch was 24.67 SD $6.46 \mathrm{~mm}$; while the distance from the proximal point of the DPUMC to the distal carpal groove was 41.4 SD $2.6 \mathrm{~mm}$. Our findings are not consistent with those reported in most previous studies. Various factors such as sample size, different measurement methodologies and phenotypic expressions of each population group evaluated can explain the variability of the DPUMC.

KEY WORDS: Median Nerve (MN); Ulnar Nerve (UN); Deep ulnar nerve branch; Deep palmar region; Communicating branch.

\section{REFERENCIAS BIBLIOGRÁFICAS}

Ahadi, T.; Raissi, G. R.; Yavari, M. \& Majidi, L. Prevalence of ulnar-tomedian nerve motor fiber anastomosis (Riché-Cannieu communicating branch) in hand: An electrophysiological study. Med. J. Islam. Repub. Iran., 30:324, 2016.

Brown, J. V. \& Landau, M. E. Sparing of the second lumbrical in a RicheCannieu anastomosis: the nearly all-ulnar hand. J. Clin. Neuromuscul. Dis., 14(4):184-7, 2013

Caetano, E. B.; Vieira, L. A.; Neto, J. J. S.; Caetano, M. F. \& Sabongi, R. G. Anastomose de Riché-Cannieu: estrutura, função e significância clínica. Rev. Bras. Ortop., 54(5):564-71, 2019.

Drake, R. L.; Wayne, V. \& Mitchell, A. W. M. Gray's Anatomy for Students. $2^{\mathrm{a}}$ ed. Philadelphia, Churchill Livingstone Elsevier. 2010.

Harness, D. \& Sekeles, E. The double anastomotic innervation of thenar muscles. J. Anat., 109(Pt. 3):461-6, 1971.

Higuet, W. B. Innervation and function of the tenar muscles. Br. Med. Bull., $7(1): 76,1943$.

Kaplan, S. Functional and Surgical Anatomy of The Hand. $3^{\mathrm{a}}$ ed. Philadelphia, J. B. Lippincott Company, 1948.

Lippert, H. Anatomía con Orientación Clínica. Madrid, Marban, 2006.

Loukas, M.; Bellary, S. S.; Tubbs, R. S.; Shoja, M. M. \& Cohen-Gadol, A. A. Deep palmar communications between the ulnar and median nerves. Clin. Anat., 24(2):197-201, 2011.

Martin, S. P.; Schauer, K. T.; Czyrny, J. J. \& Ablove, R. H. Electrophysiological findings in co mmon median-ulnar nerve interconnections and their clinical implications. J. Hand Surg. Am., 44(10):884-94, 2019.

Moore, K. L.; Agur, A. M. R. \& Dalley II, A. F. Anatomía con Orientación Clínica. $6^{\text {a }}$ ed. Barcelona, Wolters Kluwer Health, 2010.

Paraskevas, G.; Ioannidis, O. \& Martoglou, S. Cannieu-Riche anastomosis of the ulnar to median nerve in the hand: case report. Chirurgia (Bucur), 105(6):839-42, 2010.

1196
Shu, H. S.; Chantelot, C.; Oberlin, C.; Alnot, J. Y. \& Shao, H. MartinGruber communicating branch: anatomical and histological study. Surg. Radiol. Anat., 21(2):115-8, 1999.

Tamagawa, C.; Shiga, K.; Ohshima, Y.; Tokunaga, D. \& Nakagawa. M. Riche-Cannieu anastomosis and a paradoxical preservation of thenar muscles in carpal tunnel syndrome: a case report. No To Shinkei, 56(1):53-8, 2004.

Dirección para correspondencia:

Dr. Luis E. Ballesteros A. MD.

Cra. 39 \#51-31 Apto 16-01

Bucaramanga

COLOMBIA

E-mail: Iballest56@yahoo.es

Recibido: 23-02-2020

Aceptado:16-04-2020 